Original paper

\title{
Monte Carlo verification of the holder correction factors for the radiophotoluminescent glass dosimeter used by the IAEA in international dosimetry audits
}

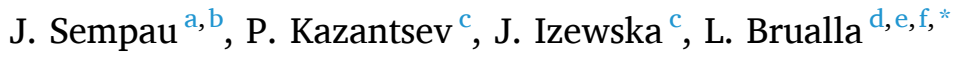 \\ ${ }^{\text {a }}$ Universitat Politècnica de Catalunya, E-08028 Barcelona, Spain \\ ${ }^{\mathrm{b}}$ Centros de Investigación Biomédica en Red en Bioingeniería, Biomateriales y Nanomedicina (CIBER-BBN), E-28029 Madrid, Spain \\ ${ }^{\mathrm{c}}$ International Atomic Energy Agency, A-1400 Vienna, Austria \\ ${ }^{\mathrm{d}}$ West German Proton Therapy Centre WPE, D-45147 Essen, Germany \\ ${ }^{\mathrm{e}}$ University of Duisburg-Essen, Faculty of Medicine, D-45147 Essen, Germany \\ ${ }^{\mathrm{f}}$ University Hospital Essen, West German Cancer Center WTZ, D-45147 Essen, Germany
}

\section{A R T I C L E I N F O}

\section{Keywords:}

Dosimetry

Radiophotoluminescent dosimetry

Correction factor

Monte Carlo

\begin{abstract}
A B S T R A C T
The International Atomic Energy Agency (IAEA), jointly with the World Health Organization (WHO), has operated a postal dosimetry audit program for radiotherapy centers worldwide since 1969. In 2017 the IAEA introduced a new methodology based on radiophotoluminescent dosimetry (RPLD) for these audits. The detection system consists of a phosphate glass dosimeter inserted in a plastic capsule that is kept in measuring position with a PMMA holder during irradiation. Correction factors for this holder were obtained using experimental methods. In this work these methods are described and the resulting factors are verified by means of Monte Carlo simulation with the general-purpose code PENELOPE for a range of photon beam qualities relevant in radiotherapy. The study relies on a detailed geometrical representation of the experimental setup. Various photon beams were obtained from faithful modeling of the corresponding linacs. Monte Carlo simulation transport parameters are selected to ensure subpercent accuracy. The simulated correction factors fall in the interval 1.005-1.008 $( \pm 0.2 \%)$, with deviations with respect to experimental values not larger than $0.2(2) \%$. This study corroborates the validity of the holder correction factors currently used for the IAEA audits.
\end{abstract}

\section{Introduction}

The International Atomic Energy Agency (IAEA), jointly with the World Health Organization (WHO), operates a worldwide postal dosimetry audit program for radiotherapy centers since 1969 [1]. Small dosimeters are sent by the IAEA to participating centers for irradiation with clinical high energy photon beams used for cancer treatment. Upon their return to the IAEA Dosimetry Laboratory, dosimeters are processed to obtain the absorbed dose to be compared with that stated by the participant. The agreement is deemed acceptable if the IAEA measured and participant stated doses differ by less than $5 \%$. Any results outside this acceptance criterion are followed-up to identify possible causes and resolve discrepancies [2]. This procedure aims at preventing adverse effects on patients originated from dosimetry errors.

Until 2016 the IAEA/WHO audit program used thermoluminescent dosimeters (TLDs) [3]. In 2017 a new radiophotoluminescent dosimetry
(RPLD) system replaced the aging IAEA TLD system [4]. Both TL and RPL dosimeters develop luminescent centers when exposed to ionizing radiation. When heated (TLD) or excited with a pulsed ultraviolet laser beam (RPLD), the luminescence centers emit light of specific wavelengths. The TL/RPL signal thus produced is proportional to the dose absorbed in the dosimeter.

Audit participants irradiate the dosimeters in a water phantom using an IAEA standard holder [3] to support the detectors in their position. This holder is absent in the experimental procedure employed by the IAEA. The influence of the IAEA holder on the TLD readings was investigated, both experimentally and analytically, by Izewska and coworkers $[5,6]$ who derived holder correction factors for the determination of absorbed dose to water. These results were compared with those obtained from Monte Carlo simulations performed by Hultqvist and co-workers [7] and good agreement was found within the associated uncertainties. The corresponding correction factors for the RPLD system

\footnotetext{
* Corresponding author.

E-mail address: Lorenzo.Brualla@uni-due.de (L. Brualla).
} 
were obtained experimentally, but no Monte Carlo verification, similar to the one done for TLDs, had been performed so far.

Although both TLDs and RPLDs use the same IAEA holder and both TLD and RPLD plastic capsules have the same external diameter fitting the holder opening, the dosimeters sensitive volumes inside the respective capsules differ in size. For the TLD a substantial part of its sensitive volume, approximately $20 \%$, is shielded by the holder walls. Consequently, holder correction factors for clinical high energy photon beams are not negligible, ranging from $1.006(25 \mathrm{MV})$ to $1.018\left({ }^{60} \mathrm{Co}\right)$ [5-7]. For the RPLD the reading signal is taken only from the central volume of the dosimeter, which fits into the holder central channel (see Section 2) and it is not directly under the holder walls "shadow". Because of the difference in dimensions and shielding of sensitive volumes of TLDs and RPLDs, their corresponding correction factors differ significantly, with those for RPLDs being closer to unity due to their relatively smaller dimensions and shielding effect.

This work presents a Monte Carlo verification of the RPLD holder correction using the general-purpose code PENELOPE for a range of photon beam qualities relevant in radiotherapy. The study relies on a detailed geometrical representation of the experimental setup to verify the experimental holder correction factors currently in use for RPLD dosimeters. Various clinically relevant high energy photon beams were obtained from faithful modeling of the corresponding linacs. Results are presented and conclusions drawn in the last part of the paper.

\section{Materials and methods}

The IAEA standard holder is a $26 \mathrm{~cm}$ long PMMA hollow cylinder with an outer diameter of $10.0 \mathrm{~mm}$ and a $2.0-\mathrm{mm}$-thick wall. It has an opening for the insertion of the dosimeter at $10 \mathrm{~cm}$ from its top, the reference depth $z_{\text {ref }}$ of measurements recommended by the TRS-398 code of practice [8]. The holder has three legs and an additional metal ring to allow precise and stable positioning in a water phantom-see Fig. 1, left.

The RPLD dosimeter considered in this study, model GD-302M from Chiyoda Technol Corporation, Japan, is made of silver activated phosphate glass of the so-called FD-7 type-see composition in Table 1, other RPLD types are described in, e.g., [9]. They are shaped as 12.0-mm-long cylinder rods, $1.5 \mathrm{~mm}$ in diameter [10]. The capsule, model M5001 produced by MISATO Precision Inc., is made of high density polyethylene (HDPE, Nipolon Hard 2000, Tosoh Corporation, Japan). It consists of a container with approximate cylindrical symmetry and with an internal 12.1-mm-long cylindrical cavity of $1.8 \mathrm{~mm}$ of diameter intended for housing the glass rod-see Fig. 1, right. Thanks to its slightly conical bevel, the capsule is tightened into the orifice that the holder has at $10 \mathrm{~cm}$ from the top. A plug completes the capsule structure and seals the cavity. The capsule and the holder are immersed in a water tank with the top of the holder lying flush with the water surface.

Some audit participants use an older version of the holder with the opening at $5 \mathrm{~cm}$ from the top. Previous recommendations for the reference conditions for different beam qualities described in the TRS277 code of practice [11], and included as an option in TRS-398, allow for both the 5 and $10 \mathrm{~cm}$ setups.

The holder correction factor, $f_{\text {hol }}$, is defined as

$f_{\text {hol }} \equiv \frac{D_{\text {wihout-holder }}}{D_{\text {with-holder }}}$

that is, as the ratio of the RPL dosimeter response without the holder to that with the holder under the same irradiation conditions. Its value depends on the beam quality and on the irradiation geometry.

\subsection{Experimental investigations}

Correction factors associated for TLDs were experimentally determined in earlier works $[5,6]$ from the interpolation of the holder effect measured with ionization chambers. Correction factors for RPLDs were also experimentally determined in a previous work [12], which used a diamond detector (60019 microDiamond from PTW, Germany) with an active diameter of $2.2 \mathrm{~mm}$ and a thickness of $1 \mu \mathrm{m}$, to measure beam profiles. The diamond detector was positioned orthogonally to the beam to allow for profile scanning through the holder opening at $z_{\text {ref }}$ with very high resolution and minimal volume averaging. Measurements were performed spanning an interval of $\pm 10 \mathrm{~mm}$ in two scanning directions and with two dosimeter orientations to encompass the length of the RPLD active volume and to avoid any bias related to the angular dependence of the detector (see [13]). The holder correction determination involved the measurement of two beam profiles in a water phantom, namely, first with the detector passing through the opening of the holder; and second, at the same depth, with the holder absent. The areas under the beam profiles within the central $6 \mathrm{~mm}$, divided by this distance, represent the dose averaged in the sensitive volume of the RPLD for both scenarios. The ratio of areas with and without the holder, corresponding to the definition given in Eq. (1), gave the holder correction factor.

This experimental methodology was applied, at a depth of $10 \mathrm{~cm}$ in water, for ${ }^{60} \mathrm{Co}, 6 \mathrm{MV}, 10 \mathrm{MV}$ and $18 \mathrm{MV}$ beams using a $10 \times 10 \mathrm{~cm}^{2}$ field at a source-to-surface distance of $100 \mathrm{~cm}$. For a depth of $5 \mathrm{~cm}$ it was carried out for ${ }^{60} \mathrm{Co}$ and $6 \mathrm{MV}$ beams under the same irradiation geometry. ${ }^{1}$ The resulting correction factors at 5 and $10 \mathrm{~cm}$ depths were fit, as functions of $\mathrm{TPR}_{20,10}$, to a straight line by least squares. It is this linear function that is being currently used for the IAEA audits.

To validate the new experimental method, the same technique was applied to reproduce the extensively validated TLD holder correction factors [5-7]. These newly calculated TLD correction factors agree with the existing ones within $0.15 \%$ [12], thus corroborating the adequacy of the methodology.

The experimental uncertainty stems mainly from two contributions, namely, (i) the variability in correction factors produced using the experimental approach described above; and (ii) the positioning of the RPLD in the holder. The component related to (i) was calculated as the average standard deviation of factors for the holders with openings at 5 $\mathrm{cm}$ and $10 \mathrm{~cm}$ and for the considered beams qualities, resulting in a relative standard uncertainty of $0.08 \%$. The component related with (ii) was considered because any potential misalignment entails an increase in the shielding of the RPLD sensitive volume by the holder walls. To estimate this component, a maximum dosimeter shift of $0.5 \mathrm{~mm}$ was assumed with a rectangular distribution for the shift probability-see [12] for details. The relative standard uncertainty related to such shift was found to be $0.12 \%$ in the worst case. The overall experimental relative standard uncertainty was evaluated as the combination of these two components in quadrature, yielding $0.14 \%$.

\subsection{Monte Carlo simulations}

Monte Carlo simulations have been performed using the PENELOPE/ penEasy system [15,16] versions 2014 (PENELOPE) and 2018-0430beta (penEasy), which has been extensively tested for dosimetry problems relevant for the present work $[7,17,21,18]$. The penEasy tally named 'energy deposition' was used to obtain the energy absorbed in the relevant spatial regions. For this section we rely on the recommendations given by the AAPM TG 268 [19] to report simulation studies.

Simulations ran in parallel in a computer cluster with 8 Intel Xeon CPU E5-2670 v3 at $2.30 \mathrm{GHz}$ clock speed, with 24 cores in each processor. The executable code for the MS Windows Server 2016 system was generated with the GNU Fortran (GCC) compiler version 4.10.0

\footnotetext{
1 Hereafter, and following a common practice in clinical radiation dosimetry (see, e.g., [14]), the tissue-phantom ratio at 20 and $10 \mathrm{~cm}$ depths $\left(\mathrm{TPR}_{20,10}\right)$ in water for a fixed source-to-detector distance and field size shall be used to specify the beam quality.
} 

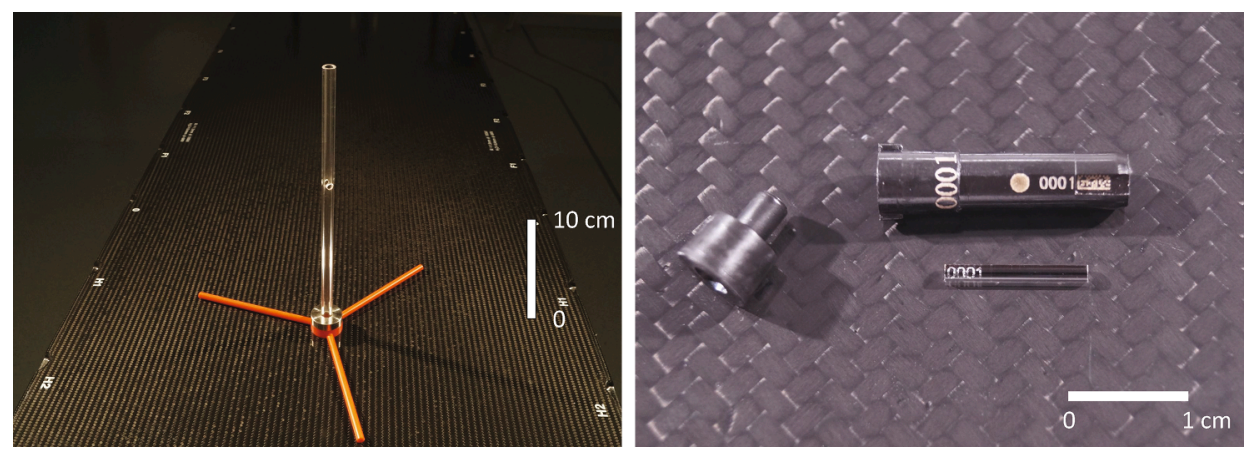

Fig. 1. IAEA holder (left) with an opening for a dosimeter at $10 \mathrm{~cm}$ from the top. Detail of the capsule and the glass rod is shown on the right.

Table 1

Materials considered in the simulation. Compositions are in percentage by weight, mass densities in $\mathrm{g} / \mathrm{cm}^{3}$ and mean excitation energies, $\mathrm{I}$, in $\mathrm{eV}$.

\begin{tabular}{cclc}
\hline Material & Composition & Mass density & $I$ \\
\hline FD-7 & $\mathrm{Ag}(0.17 \%), \mathrm{Al}(6.12 \%)$ & & \\
& $\mathrm{Na}(11.0 \%), \mathrm{P}(31.55 \%)$ & 2.61 & 124.5 \\
HDPE & $\mathrm{O}(51.16 \%)$ & & \\
PMMA & $\mathrm{C}_{2} \mathrm{H}_{4}$ & 0.96 & 57.4 \\
Liquid water & PENELOPE database & 1.19 & 74.0 \\
Dry air & $\mathrm{H}_{2} \mathrm{O}$ & 1.00 & 78.0 \\
& PENELOPE database & $1.205 \times 10^{-3}$ & 85.7 \\
\hline
\end{tabular}

20140629 using the optimization option "-O”. Typical simulation time, using all processors, was in the order of $130 \mathrm{~h}$ to attain relative standard uncertainties of $\sim 0.12 \%$.

\subsubsection{Geometry model}

The modeling of the holder and the capsule was derived from detailed blueprints provided by the manufacturer. The essential information regarding shape and dimensions was summarized above.

Fig. 2 shows the simulation model of the capsule, including the glass rod, inserted in its holder. Color blue represents the PMMA material and green corresponds to the HDPE capsule. The FD-7 glass rod is divided into two distinct regions, namely, the one depicted orange and the purple. The latter corresponds to the active volume whereas the former, due to the details of the reading process, is considered inactive for detection purposes. In the Monte Carlo simulations only the energy deposited in the active, i.e. purple, region is tallied. The materials considered and their most relevant properties are shown in Table 1.

\subsubsection{Radiation sources}

Point-like sources emitting photon beams of the considered qualities were defined. Linac beam qualities (4, 6, 10, 15 and $18 \mathrm{MV}$ ) were modeled using the spectra published in the PRIMO project website. ${ }^{2}$ The procedure used for obtaining these energy distributions is explained by Brualla et al. [20].

\subsubsection{Simulation parameters}

General-purpose codes rely on a number of user-defined parameters to define the energy cutoffs, at which particles are stopped and their remaining energy is locally absorbed, and the configuration of the socalled condensed simulation schemes, approximate algorithms that serve to speed up the simulation of charged particle transport. The values adopted for these parameters determine the trade-off between speed and accuracy in each case.

In the case of PENELOPE this trade-off has been amply studied for potentially problematic geometries like those of air-filled ion chambers

\footnotetext{
$\overline{2}$ https://www.primoproject.net/primo/EnergySpectra.
}

[17]. Fortunately for the purpose of the present study, the simulation of dense and relatively homogenous detectors (that is, not small gas cavities with dense walls like in an ion chamber) is not among the problematic cases. In addition, PENELOPE exhibits a remarkable stability with respect to the selected parameter values so that their precise value is not critical, provided certain reasonable constraints are observed (see $[17,15]$ Section 4.4.1).

The parameter values chosen for this study are detailed in Table 2 . They have been selected according to the experience gained in the simulation of ion chambers and other dosimetric devices (see e.g. $[21,22])$. Their interpretation is as follows: $E_{\text {abs }}(\gamma)$ and $E_{\text {abs }}\left(e^{ \pm}\right)$are the cutoff (kinetic, in the $\mathrm{e}^{ \pm}$case) energies at which simulation is terminated and the particle remaining energy is locally absorbed; $C_{1}$ and $C_{2}$ are the electron average angular deflection and the maximum fractional energy loss allowed in one step, respectively; $W_{\mathrm{cc}}$ and $W_{\mathrm{cr}}$ are the energies that separate hard from soft events for inelastic and bremsstrahlung interactions, respectively; and $s_{\max }$ is the maximum allowed step length of charged particles. The reader is referred to the PENELOPE manual [15] for a more detailed description of these parameters.

To ascertain the correctness of the chosen parameters one case was simulated with more demanding values, namely, $E_{\mathrm{abs}}\left(e^{ \pm}\right)$and $W_{\mathrm{cc}}$ were reduced by a factor of one hundred, whereas $C_{1}, C_{2}$ and $W_{\mathrm{cr}}$ were reduced by a factor of ten. The results obtained were compatible with those yielded by the equivalent simulation with larger parameters at the uncertainty subpercent level sought in the present study.

It should be remarked that two different water materials are defined. The one termed "water skin" is used for water in close proximity with the capsule, where more refined conditions are adopted for better accuracy. Farther away electron transport is not so critical, since the direction of flight becomes randomized after relatively short distances. The same strategy was followed with the PMMA conforming the holder. The thickness of the skin region around the capsule was set to $0.5 \mathrm{~cm}$ based on the continuous slowing down approximation (CSDA) range of the electrons at the relevant energies. The small amount of air trapped between the glass rod and the capsule was simulated using the same transport parameters as those employed for the rod and the capsule themselves, while the air above the water tank used less stringent values. No other variance-reduction techniques were used.

\section{Results}

The linear fits obtained by Kazantsev and co-workers [12], resulting from the experimental procedure described in Section 2.1, were as follows

$f_{\text {hol }}^{(5 \mathrm{~cm})}=-0.0005 \mathrm{TPR}_{20,10}+1.0035$

and

$f_{\text {hol }}^{(10 \mathrm{~cm})}=-0.0035 \mathrm{TPR}_{20,10}+1.0085$ 

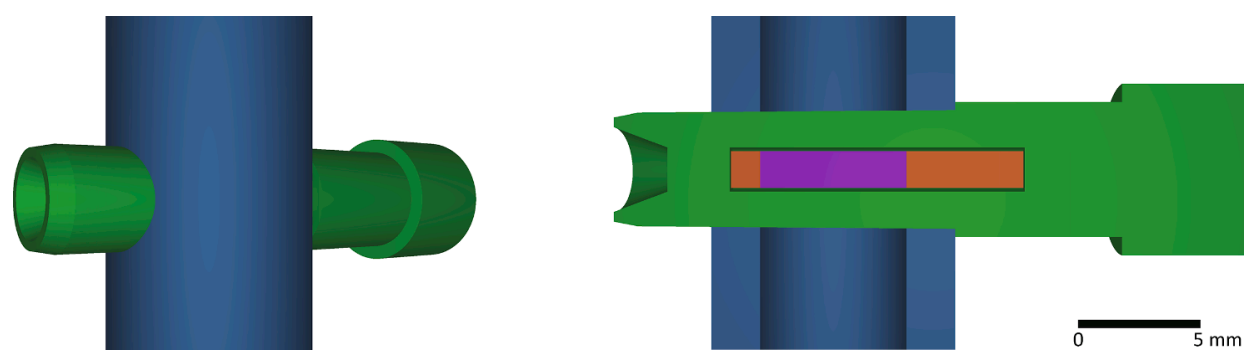

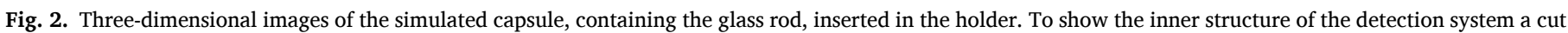

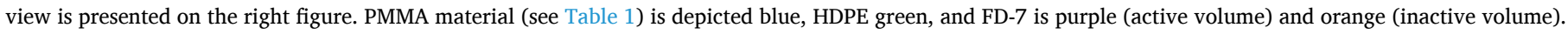
Note that the glass rod is divided in two regions for tallying purposes (see text for details).

Table 2

Simulation parameters used in the simulations. $E_{\mathrm{abs}}(\gamma), E_{\mathrm{abs}}\left(e^{ \pm}\right), W_{\mathrm{cc}}$ and $W_{\mathrm{cr}}$ are given in keV. $s_{\max }$ is given in $\mathrm{cm} . C_{1}$ and $C_{2}$ are dimensionless.

\begin{tabular}{cccccccc}
\hline Material & $E_{\mathrm{abs}}(\gamma)$ & $E_{\mathrm{abs}}\left(e^{ \pm}\right)$ & $C_{1}$ & $C_{2}$ & $W_{\mathrm{cc}}$ & $W_{\mathrm{cr}}$ & $s_{\max }$ \\
\hline FD-7 & 10 & 100 & 0.1 & 0.1 & 100 & 10 & 0.01 \\
HDPE & 10 & 100 & 0.1 & 0.1 & 100 & 10 & 0.01 \\
PMMA & 10 & 500 & 0.1 & 0.1 & 500 & 10 & $\infty$ \\
PMMA skin & 10 & 100 & 0.1 & 0.1 & 100 & 10 & $\infty$ \\
Water & 10 & 500 & 0.1 & 0.1 & 500 & 10 & $\infty$ \\
Water skin & 10 & 100 & 0.1 & 0.1 & 100 & 10 & $\infty$ \\
Air above tank & 10 & 1000 & 0.1 & 0.1 & 1000 & 10 & $\infty$ \\
Air in capsule & 10 & 100 & 0.1 & 0.1 & 100 & 10 & 0.01 \\
\hline
\end{tabular}

for depths 5 and $10 \mathrm{~cm}$, respectively. The coefficient of determination was $r^{2}=0.979$ for the $10 \mathrm{~cm}$ case; the $5 \mathrm{~cm}$ case involved two beam qualities only, thus $r^{2}$ is trivially unity. The relative standard uncertainty for $f_{\text {hol }}$ was estimated to be $0.14 \%$.

Simulation results are given in numerical form in Table 3 and in graphical format, at $10 \mathrm{~cm}$ depth, in Fig. 3 together, for comparison, with the corresponding values obtained from the experimental linear fitting functions.

\section{Discussion and conclusions}

Experimental correction factors exhibit, at the $0.1 \%$ uncertainty level achieved, an imperceptible beam quality dependence for the range of photon energies investigated, attaining a constant value of 1.006(1) at $10 \mathrm{~cm}$ depth. The datum at $5 \mathrm{~cm}$ depth is smaller, 1.003(1), although within two standard deviations (95\% confidence level) compatible with the value at $10 \mathrm{~cm}$. Similar conclusions can be drawn from the simulation data regarding their variability with respect to beam quality and with respect to depth, with no clear trend in the explored energy and depth ranges.

It is interesting to point out that differences between correction factors at 5 and $10 \mathrm{~cm}$ depths are to be expected, with values farther from unity at deeper positions in the water phantom. Indeed, PMMA has a total attenuation coefficient that is, approximately, 16\% larger than that of water for a wide energy range, from $0.3 \mathrm{MeV}$ up to $3 \mathrm{MeV}$, being still $13 \%$ larger than that of water at $10 \mathrm{MeV}$. This difference stems mainly from the different mass densities, with that of PMMA being nearly $19 \%$ larger. At greater depths, and for any given beam quality, a higher proportion of scattered low energy photons travel sideways across the PMMA tube, thus making more relevant the difference between the energy averaged attenuation coefficients for the with-holder case with respect to the reference water-only case. The fact that this difference cannot be clearly established from measurements, nor from simulations, gives an upper bound to its value based on the uncertainties of both data sets, namely, around $0.2 \%$.

The relative constancy of the ratio of attenuation coefficients PMMA/water with photon energy (around 1.16, as discussed above) is also the main cause of the little variation of $f_{\text {hol }}$ with beam quality,
Table 3

Comparison between simulated (sim) and experimental fitting line (exp) RPLD holder correction factors for the different beam qualities $\mathrm{TPR}_{20,10}$ considered in the simulation. Standard (one $\sigma$ ) uncertainties are indicated within parenthesis. The relative deviation (in \%) of simulation data with respect to the experimental values is indicated in the last column.

\begin{tabular}{cccccc}
\hline Quality & $z_{\text {ref }}$ & $\mathrm{TPR}_{20,10}$ & $f_{\text {hol }}^{\text {(sim) }}$ & $f_{\text {hol }}^{(\text {exp }}$ & Deviation \\
\hline $6 \mathrm{MV}$ & 5 & $0.6553(2)$ & $1.0052(08)$ & $1.0032(14)$ & $+0.2 \%$ \\
$4 \mathrm{MV}$ & 10 & $0.6335(3)$ & $1.0070(20)$ & $1.0063(14)$ & $+0.1 \%$ \\
$6 \mathrm{MV}$ & 10 & $0.6553(2)$ & $1.0061(20)$ & $1.0062(14)$ & $+0.0 \%$ \\
$10 \mathrm{MV}$ & 10 & $0.7299(2)$ & $1.0053(20)$ & $1.0060(14)$ & $-0.1 \%$ \\
$15 \mathrm{MV}$ & 10 & $0.7518(2)$ & $1.0067(19)$ & $1.0059(14)$ & $+0.1 \%$ \\
$18 \mathrm{MV}$ & 10 & $0.7762(2)$ & $1.0081(16)$ & $1.0058(14)$ & $+0.2 \%$ \\
\hline
\end{tabular}

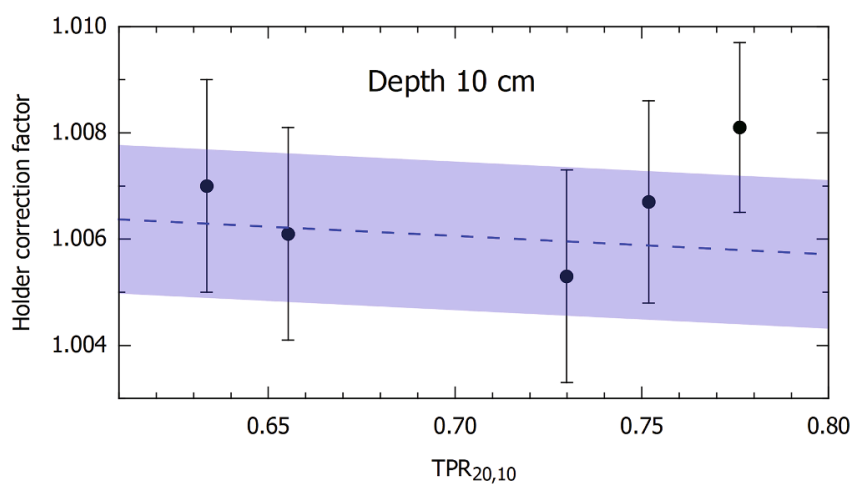

Fig. 3. Comparison between simulated holder correction factors at $10 \mathrm{~cm}$ depth (black dots) and the linear fit obtained from experimental values that is currently used in the IAEA audits (blue dashed line). The bluish band covers the region within one standard uncertainty $( \pm 0.14 \%)$ of the experimental values. Simulation uncertainty bars are also at one $\sigma$ level. Uncertainties associated to the simulated $\mathrm{TPR}_{20,10}$ 's are smaller than symbol size. From left to right the simulated beam qualities are $4 \mathrm{MV}, 6 \mathrm{MV}, 10 \mathrm{MV}, 15 \mathrm{MV}$ and $18 \mathrm{MV}$.

virtually undetectable at the attained uncertainty level of this study.

Overall, the agreement between experiments and simulations is well within the $\sim 0.1 \%$ uncertainty level of the experimental data. This fact reassures the confidence on the holder correction factors being applied for the RPLD audits since 2017.

\section{Declaration of Competing Interest}

JS and LB are consultants of the IAEA. PK is employee of the IAEA. JI is a retired employee of the IAEA. 


\section{Acknowledgements}

JS thanks the Spanish Ministerio de Ciencia, Innovación y Universidades, project FPA2017-83946-C2-2-P and the H2020 European Joint Programme Concert, project 003-2017-PODIUM for partial financial support. LB is thankful to the HARMONIC project. The HARMONIC project (Health effects of cArdiac fluoRoscopy and MOderN radIotherapy in paediatriCs) has received funding from the Euratom research and training programme 2014-2018 under grant agreement No 847707.

\section{References}

[1] Izewska J, Bokulic T, Kazantsev P, Wesolowska P, van der Merwe D. 50 Years of the IAEA/WHO postal dose audit programme for radiotherapy: what can we learn from 13756 results? Acta Oncol 2020;59:495-502. https://doi.org/10.1080/ 0284186X.2020.1723162.

[2] Kry SF, Peterson CB, Howell RM, Izewska J, Lye J, Clark CH, Nakamura M, Hurkmans C, Alvarez P, Alves A, Bokulic T, Followill D, Kazantsev P, Lowenstein J, Molineu A, Palmer J, Smith SA, Taylor P, Wesolowska P, Williams I. Remote beam output audits: a global assessment of results out of tolerance. Phys Imag Radiat Oncol 2018;7:39-44. https://doi.org/10.1016/j.phro.2018.08.005.

[3] Izewska J, Andreo P. The IAEA/WHO TLD postal programme for radiotherapy hospitals. Radiother Oncol 2000;54:65-72.

[4] Wesolowska PE, Cole A, Santos T, Bokulic T, Kazantsev P, Izewska J. Characterization of three solid state dosimetry systems for use in high energy photon dosimetry audits in radiotherapy. Radiat Meas 2017;106:556-62.

[5] Izewska J, Novotny J, Van Dam J, Dutreix A, Van Der Schueren E. The influence of the IAEA standard holder on dose evaluated from TLD samples. Phys Med Biol 1996;41:465-73.

[6] Izewska J, Georg D, Bera P, Thwaites D, Arib M, Saravi M, Sergieva K, Li K, Yip FG, Mahant AK, Bulski W. A methodology for TLD postal dosimetry audit of highenergy radiotherapy photon beams in non-reference conditions. Radiother Oncol 2007;84:67-74.

[7] Hultqvist M, Fernández-Varea JM, Izewska J. Monte Carlo simulation of correction factors for IAEA TLD holders. Phys Med Biol 2010;55:N161-6.

[8] IAEA. Absorbed dose determination in external beam radiotherapy: an international code of practice for dosimetry based on standards of absorbed dose to water. Technical Report TRS 398, International Atomic Energy Agency; 2000.

[9] Mizuno H, Kanai T, Kusano Y, Ko S, Ono M, Fukumura A, Abe K, Nishizawa K, Shimbo M, Sakata S, Ishikura S, Ikeda H. Feasibility study of glass dosimeter postal dosimetry audit of high-energy radiotherapy photon beams. Radiother Oncol 2008; 86:258-63.

[10] Araki F, Moribe N, Shimonobou T, Yamashita Y. Dosimetric properties of radiophotoluminescent glass rod detector in high-energy photon beams from a linear accelerator and cyber-knife. Med Phys 2004:31:1980-6.

[11] IAEA. Absorbed Dose Determination in Photon and Electron Beams; An International Code of Practice, Technical Report 277, IAEA, Vienna; 1987.

[12] Kazantsev P, Wesolowska P, Santos T, Szegedi D, Izewska J. Experimental determination of the standard IAEA holder correction factor for RPLD postal dose audit applications. In: Petrovic B, Ciraj-Bjelac O, editors. Proceedings of the 8th Alpe-Adria Medical Physics Conference, Serb. Assoc. Med. Phys., Soc. Rad. Prot. Serb. Monten., Vinca Inst. Nuc. Sci., Borislav Grubor, Novi Sad, Serbia; 2017. p. $162-8$.

[13] IAEA. Dosimetry of Small Static Fields Used in External Beam Radiotherapy, Technical Report TRS 483, International Atomic Energy Agency; 2017.

[14] Andreo P, Burns DT, Nahum AE, Seuntjens J, Attix FH. Fundamentals of ionizing radiation dosimetry. Weinheim: Wiley-VCH; 2017.

[15] Salvat F. PENELOPE-2014: A code system for Monte Carlo simulation of electron and photon transport. OECD Nuclear Energy Agency, Issy-les-Moulineaux, France; 2015. Available in pdf format at http://www.oecd-nea.org.

[16] Sempau J, Badal A, Brualla L. A PENELOPE-based system for the automated Monte Carlo simulation of clinacs and voxelized geometries-application to far-from-axis fields. Med Phys 2011;38:5887-95. https://doi.org/10.1118/1.3643029.

[17] Sempau J, Andreo P. Configuration of the electron transport algorithm of PENELOPE to simulate ion chambers. Phys Med Biol 2006;51:3533-48.

[18] Erazo F, Brualla L, Lallena A. Computation of the electron beam quality kQ, Q0 factors for the NE2571, NE2571A and NE2581A thimble ionization chambers using PENELOPE. Phys Med 2017;38:76-80. https://doi.org/10.1016/j. ejmp.2017.05.053.

[19] Sechopoulos I, Rogers DWO, Bazalova-Carter M, Bolch WE, Heath EC, McNittGray MF, Sempau J, Williamson JF. RECORDS: improved Reporting of montE CarlO RaDiation transport Studies: Report of the AAPM Research Committee Task Group 268. Med Phys 2018;45:e1/5.

[20] Brualla L, Rodriguez M, Sempau J, Andreo P. PENELOPE/ PRIMO-calculated photon and electron spectra from clinical accelerators. Radiat Oncol 2019;14:1-10. https://doi.org/10.1186/s13014-018-1186-8.

[21] Erazo F, Brualla L, Lallena A. Electron beam quality kQ, Q0 factors for various ionization chambers: A Monte Carlo investigation with PENELOPE. Phys Med Biol 2014;59:6673-91. https://doi.org/10.1088/0022-3727/59/21/6673.

[22] Goma C, Andreo P, Sempau J. Monte Carlo calculation of beam quality correction factors in proton beams using detailed simulation of ionization chambers. Phys Med Biol 2016;61:2389-406. https://doi.org/10.1088/0031-9155/61/6/2389. 